\title{
Participación de profesores en un ambiente de formación online. \\ Un estudio en modelación matemática
}

\section{Teacher's participation in Online Education Environments. A Research in Mathematical Modelling}

\author{
Mónica Marcela Parra-Zapata, Paula Andrea Rendón-Mesa, \\ María Camila Ocampo-Arenas, Jonathan Sánchez-Cardona \\ Juan Fernando Molina-Toro, Jhony Alexander Villa-Ochoa ${ }^{1}$
}

\begin{abstract}
Resumen. Este artículo analiza la participación de un conjunto de profesores en una sesión de modelación matemática en un espacio de formación online. La participación se estudió a partir de la visibilidad, la interactividad, las interacciones y las contribuciones. El análisis se centró en las acciones, los roles y las voces de los docentes cuando se comprometieron con las maneras de usar la modelación y reflexionaron en torno a su conocimiento de este proceso. El estudio fue cualitativo. Se propusieron tareas en colectivos de profesores de manera sincrónica y asincrónica, para lo cual se usaron las plataformas Moodle y WizIQ, y la herramienta Google Drive. A partir de este estudio se reconocieron características de una comprensión más amplia de la participación que trasciende la atención en la visibilidad, y se enfoca en las acciones, las relaciones entre los sujetos, los recursos y el contenido involucrado en esa participación. Se concluye que para promover una participación con mayor contenido en ambientes online, es preciso generar diferentes experiencias con relación al conocimiento.
\end{abstract}

Fecha de recepción: 26 de abril de 2016. Fecha de aceptación: 30 de enero de 2018

1 Universidad de Antioquia, Colombia, monica.parra@udea.edu.co; paula.rendon@udea.edu.co; camila. ocampo@udea.edu.co; jonathan.sanchezc@udea.edu.co; juan.molinat@udea.edu.co; jhony.villa@udea.edu.co 
Palabras clave: participación; modelación matemática; ambientes online.

\begin{abstract}
This paper analyzes the participation of a group of teachers in a session about mathematical modelling in an online training environment. The participation was studied based on visibility, interactivity, interactions and contributions. Analysis focused on recognizing the actions, roles and voices of teachers when they engaged in the ways of using mathematical modelling and generated reflections around their knowledge of this process. The research was qualitative. Tasks were designed and developed in groups of teachers in synchronous and asynchronous ways, using Moodle and WizlQ platforms, and Google Drive tools. From this research, they were recognized characteristics through a broader understanding of participation that transcend the attention in the visibility, and it is focused on the actions, relations between the subjects, resources and content involved in that participation. The conclusion is that to promote participation with greater content in online environments, it is necessary to generate different knowledge related experiences to revitalize the training processes.
\end{abstract}

Keywords: Participation; Mathematical modelling; Online environments.

\title{
1. INTRODUCCIÓN
}

La investigación acerca de los recursos y la formación de profesores arroja resultados importantes para caracterizar el conocimiento del docente en relación con la tecnología (Crompton, 2015; Larkin et al., 2012), los usos de la tecnología que hacen los profesores de matemáticas (Agyei y Voogt, 2011) y los ambientes, las modalidades y los recursos utilizados en su formación (Sacristán et al., 2013). Frente a este último tema se plantea la vinculación de tres componentes: la consolidación de comunidades de aprendizaje o de práctica (Sacristán et al., 2013); la conformación de colectivos de docentes en donde los medios no son solo auxiliares sino partes constitutivas de la producción de conocimientos (Souto y Borba, 2016; Villa-Ochoa et al., 2014), y la configuración de ambientes E-Learning o B-Learning (Javaroni y Zampieri, 2016; Rendón-Mesa et al., 2016).

En Colombia, al igual que en otros países de Latinoamérica, existe una diversidad de enfoques y necesidades de formación de profesores de 
matemáticas; también hay un creciente interés, posibilidades de financiación y acceso a programas de posgrado dedicados a formar docentes en esta área. Sin embargo, las principales modalidades de formación en estos programas son face-to-face (Guacaneme-Suárez et al., 2017). A pesar de que en Colombia existen instituciones que ofrecen programas de posgrado en modalidades mixtas (B-Learning) o totalmente a distancia online, los fundamentos bajo los cuales son estructurados estos programas y las maneras en que se proponen atender las diversas necesidades de formación de los profesores de matemáticas es aún difusa.

En investigaciones recientes de formación de profesores online (Borba et al., 2016; Silverman, 2012; Fernández et al., 2012) hay un reconocimiento explícito a su participación en estos ambientes. El estudio de Silverman (2012) explora la relación entre la participación de los docentes en las discusiones en línea y el desarrollo de su conocimiento de los contenidos matemáticos para la enseñanza. Sus hallazgos indican que la participación no se correlacionó con el desarrollo del conocimiento matemático para la enseñanza, sino que evidenció una relación entre la prominencia de un individuo en sesiones de colaboración y el desarrollo de tal conocimiento. Por su parte, Fernández et al. (2012) mencionan la participación como medio para involucrar a los futuros profesores en discusiones a través de las cuales se promueve su capacidad para interpretar el pensamiento matemático de sus estudiantes.

En los estudios mencionados se observa interés por promover la participación como un medio para el desarrollo de un conocimiento del contenido o para fomentar discusiones, reflexiones y capacidades en los docentes de matemáticas. Sin embargo, el interés por la participación de los profesores en ambientes online como objeto de estudio no aparece explícito en estos trabajos; es decir, la investigación que procure la identificación y caracterización de maneras de participación aún es un tema abierto en el campo de la Educación Matemática online.

Con base en lo anterior, en este trabajo se analiza la participación en un espacio de formación online para profesores de matemáticas adscritos a un programa de maestría profesionalizante. En particular, estudia las características de la participación de los estudiantes (profesores en ejercicio) a partir de sus acciones, roles y voces en torno al conocimiento. El interés de analizar la participación se fundamenta en la relevancia de los ambientes que promueven aprendizajes colaborativos y cómo se generan las dinámicas entre estudiantes, profesores y el conocimiento. Reconocer la participación, en el sentido propuesto, 
se torna importante en el desarrollo profesional de los docentes de matemáticas, pues se convierte en un medio a través del cual se promueven discusiones, reflexiones y análisis en pro del desarrollo del conocimiento matemático.

\section{CONSIDERACIONES TEÓRICAS}

La formación para profesores en un ambiente online sugiere el reconocimiento de nuevas herramientas, escenarios y actuaciones, tanto de quienes realizan el papel de formadores como de aquellos que asumen el proceso formativo. En una investigación reciente Flores et al. (2014) reportaron los hallazgos de un estudio documental en la formación de docentes de matemáticas en ambientes online. Los autores señalan que existe un interés común en aspectos del conocimiento de los profesores de matemáticas a través del uso de entornos en línea; asimismo, reconocieron dos objetos de estudio: i) El análisis de las interacciones entre los profesores en el ambiente online, y ii) El desarrollo profesional de los docentes.

\subsection{LA PARTICIPACIÓN EN AMBIENTES ONLINE}

La participación ha sido objeto central en los estudios de Álvarez (2010), Malinen (2015), Parra-Zapata y Villa-Ochoa (2016), Amichai-Hamburger et al. (2016) y Parra-Zapata et al. (2017), entre otros. Malinen (2015) resalta que en la investigación internacional la participación online se ha enfocado típicamente en analizar la cantidad de veces que los usuarios aportan a las discusiones online (visibilidad) y propone que, en lugar de centrarse en dicho criterio, se preste atención a la calidad de la actividad en términos de su relevancia para la comunidad. La autora pone de relieve la necesidad de trascender la noción de participación activa -entendida como la cantidad de conexiones- y recomienda que en los ambientes online se recurra a diseños que posibiliten interacción de los participantes.

En coherencia con el trabajo de Parra-Zapata et al. (2017), y con el ánimo de identificar y promover factores para la formación de profesores en ambientes online, en este estudio se identifica y caracteriza la participación a partir de cuatro componentes, a saber: la visibilidad (Malinen, 2015), la interactividad (Rost, 2004), las interacciones y las contribuciones (Parra-Zapata y Villa-Ochoa, 
2016). Se describen a continuación las cuatro componentes de la participación, las cuales no son disjuntas, es decir, en un ambiente escolar se presentan articuladas y simultáneamente, e incluso, en algunos casos, los límites entre ellas son difusos.

La visibilidad se reconoce en la cantidad de veces que el usuario se conecta, la duración de las mismas y la motivación que presenta para realizar las intervenciones (Malinen, 2015). Sin embargo, es importante concebir la visibilidad en términos de la calidad y el contenido de la participación, es decir, de los aportes o las reflexiones que dan cuenta de apropiaciones teóricas o prácticas que, a la vez, posibilitan el surgimiento de otras ideas.

La interactividad se entiende como la relación que establecen una o varias personas con el entorno digital (Rost, 2004). En este sentido, el emisor es el sujeto y el receptor es la máquina o plataforma que media y hace posible el proceso formativo. Esta componente de la participación se percibe en el papel activo del usuario cuando selecciona las informaciones requeridas. Al mismo tiempo, se reconoce como la capacidad técnica de conceder el máximo de posibilidades de comunicación, intervención y actuación con los recursos del entorno digital, de tal manera que se incrementen las intervenciones del usuario en el desarrollo del conocimiento y en el entorno mismo (Danvers, 1994). De acuerdo con ello, la interactividad se reconoce en las posibilidades que enmarquen las características de los contenidos y las propiedades del medio o recurso tecnológico para generar diálogos, reflexiones y aportes.

Las interacciones se asumen como una acción social que un sujeto lleva a cabo con otro u otros sujetos. En tal sentido, es el acto de comunicar, discutir y compartir con los demás puntos de vista, ideas, conocimientos, reflexiones, sentimientos, hallazgos y posturas con respecto a un objeto de estudio. No consiste simplemente en ofrecer un mensaje o una respuesta, sino en una serie de discusiones coherentes entre estudiantes, con o sin la participación del profesor, para lo cual es necesario que la actividad esté orientada a fomentar el análisis de diversos puntos de vista y la toma de posición al respecto (Parra-Zapata y VillaOchoa, 2016). En las interacciones se detectan las relaciones entre los sujetos, la reflexión acerca del conocimiento, la manifestación de sentimientos, las discusiones con relación a la respuesta de los compañeros, la toma de posición respecto a una temática de debate, la relación con el otro, o el intercambio de información. Como se argumentará más adelante, cuando se pone la atención en las relaciones sujeto-contenido, también se reconocen otras maneras de interacción; principalmente, aquellas en las que se produce conocimiento acerca del contenido. 
Las contribuciones son los aportes a las discusiones y a la actividad realizada. Los aportes van más allá de "estoy de acuerdo" o "no me parece" y se vinculan con los argumentos y demás participantes, la ayuda o colaboración con los otros, la exploración de saberes y la construcción colectiva de significados. Dichas acciones agregan valor a lo discutido, ayudan a otros a expresar sus pensamientos y a explorar nuevas áreas (Parra-Zapata y Villa-Ochoa, 2016).

\subsection{LA FORMACIÓN DE PROFESORES EN Y PARA LA MODELACIÓN MATEMÁTICA EN AMBIENTES ONLINE}

Los ambientes online son espacios que promueven la producción de conocimiento en y para la Educación Matemática, a partir de diferentes maneras de participación, de unos propósitos de formación para profesores y reflexiones acerca de sus prácticas. En correspondencia con ello, se asume una visión de formación en la que es importante que los estudiantes se enfrenten a experiencias en las cuales produzcan conocimientos y establezcan acciones para integrarlas a sus contextos de desempeño profesional.

Considerar las prácticas de los docentes en ambientes online pone de manifiesto, entre otros asuntos, el reconocimiento de las posibilidades o alternativas metodológicas e investigativas que hacen parte de la formación matemática. En este artículo se consideró la modelación matemática como una alternativa para promover, en los profesores, maneras distintas de participación; desarrollar actitudes creativas y críticas, así como capacidades hacia la resolución de problemas alrededor de las matemáticas. El diseño del ambiente online tuvo en cuenta que el conocimiento del docente, para la integración de la modelación matemática en sus prácticas escolares, no se agota en las clásicas características de un conocimiento del contenido matemático, de las estrategias pedagógicas y la multiplicidad de maneras en que se puede desarrollar el pensamiento de los estudiantes sino que, más allá de ello, deben considerarse las acciones en correspondencia con sus prácticas y las maneras en que las interpretan (Doerr y Lesh, 2011).

La modelación matemática ha sido uno de los ejes fundamentales de las investigaciones en educación online en Brasil (Galleguillos, 2016; Borba et al., 2014). Borba et al. (2014) reportaron que en las experiencias de formación online debe generarse como un medio para promover la comunicación entre los participantes y posibilitar maneras de experimentación. La experiencia de los autores 
evidencia que la educación online trasciende las aulas y que en el desarrollo de repositorios y plataformas, los profesores, estudiantes e investigadores pueden interactuar, con el fin de desarrollar productos en el área. Por su parte, Galleguillos (2016) presenta los avances de un estudio realizado con docentes en ejercicio en un curso de extensión; entre las temáticas del curso está la modelación matemática. Los participantes se enfrentaron a un problema de modelación relacionado con un tema que fue de interés para el grupo.

Con base en las experiencias mencionadas, en este artículo se describe una sesión de trabajo de cuatro horas en la cual se discutió y reflexionó en torno a la modelación matemática, con el fin de promover la participación de los profesores. En el siguiente apartado se presentan los aspectos metodológicos de la investigación, al igual que las estrategias y métodos utilizados en el curso.

\section{ASPECTOS METODOLÓGICOS}

\subsection{LA METODOLOGÍA Y EL CONTEXTO}

Conforme se mencionó, esta investigación se fundamentó en el análisis de la participación de un colectivo de docentes en un ambiente online. Poner la atención en este tipo de fenómenos requiere que los investigadores centren su mirada en las actuaciones y diálogos de cada participante, y el rol que juegan en cada momento de la experiencia; por esta razón se adoptó una metodología cualitativa. En relación con los ambientes online, la investigación cualitativa en educación y en ciencias sociales impone nuevos desafíos frente a la naturaleza y maneras de obtención de los datos, condiciones éticas y los métodos mismos de investigación (Gerber et al., 2016; Andrews y Haythornthwaite, 2007).

En el estudio participaron 17 profesores que, a su vez, eran estudiantes de posgrado en Educación Matemática de dos universidades en Brasil y Colombia (nueve brasileños y ocho colombianos). Los docentes formaban parte de un curso diseñado en colaboración por dos grupos de investigación de los respectivos países. Dado que la ubicación geográfica y la lengua materna de los participantes eran diferentes, se consolidaron cinco grupos de trabajo para el desarrollo de tareas de modelación de manera sincrónica y asincrónica. En las sesiones asincrónicas, la conformación de estos grupos cumplió un rol fundamental para el uso y la preparación previa en herramientas participativas (Google Drive, Skype, Google Hangout, etc.), así como para proporcionar un primer 
acercamiento al desarrollo y críticas de tareas de modelación matemática. Los grupos estuvieron configurados de tal manera que cada equipo tuviera participantes de ambas nacionalidades. Asimismo, las dinámicas de cada equipo atendieron a las observaciones de Gerber et al. (2016) en el sentido de considerar los aspectos que emergían de los cuestionamientos y las necesidades de los participantes, y no para atender a una estructura rígida y prescrita por los profesores orientadores del curso.

En coherencia con las consideraciones de Gerber et al. (2016), los recursos disponibles para el curso promovieron diferentes maneras de participación de los docentes, es decir, no fueron usados de una manera domesticada para replicar metodologías transmisionistas ni expositivas en donde la comunicación se ofrece únicamente en la vía profesor-estudiante, sino que se utilizaron todos los recursos disponibles para promover multi-diálogos e interacciones en pequeños grupos, y de éstos con los coordinadores del curso y el colectivo en general. Como recursos adicionales se dispuso de WizIQ, Moodle, Google Drive y Youtube.

\subsection{EL CURSO Y EL DISEÑO DE LA SESIÓN DE MODELACIÓN}

El curso se implementó a lo largo del segundo semestre de 2015 y estuvo dedicado al estudio de las temáticas: educación a distancia online, tecnologías digitales, visualización, formación de profesores, modelación matemática y evaluación en matemáticas. Para este artículo se estudió el diseño y desarrollo de la temática modelación matemática, la cual contó con una sesión sincrónica (4 horas) y dos sesiones asincrónicas (una previa y otra posterior a la sesión sincrónica).

El diseño de la sesión de modelación se fundamentó en el trabajo de Parra-Zapata et al. (2017) y, conforme sugieren estos autores, en la sesión asincrónica previa los estudiantes analizaron experiencias de modelación reportadas en dos videos. Las reflexiones y las críticas de estos videos se convirtieron en insumo para alimentar el debate en torno a la temática de la sesión; para ello se dispuso de un foro y dos Wikis, una para los participantes interesados en la modelación matemática como un recurso en sus prácticas de profesores y otra para los interesados en la investigación en modelación en Educación Matemática.

En la sesión asincrónica previa también se propuso una tarea de modelación para cada grupo de trabajo. La tarea contenía un conjunto de cuestionamientos acerca de las características de la modelación, las posibilidades, las limitaciones y ajustes que podría recibir en caso de que se pudiera implementar; además, 
cuestionaba la propia práctica en relación con el uso de la modelación. En este trabajo asincrónico cada equipo de trabajo contó con el acompañamiento de un profesor orientador. Una semana después de realizar la tarea de modelación matemática se desarrolló la sesión sincrónica, la cual se diseñó para que los estudiantes lograran tener una visión acerca de las diferentes maneras de hacer modelación.

La sesión sincrónica se fundamentó en tres ejes temáticos. El primero se relacionó con aspectos teóricos de la modelación matemática (como práctica, perspectiva metodológica y ambiente de investigación, entre otros). Asimismo, para promover la reflexión frente a las tareas previamente desarrolladas de manera asincrónica y para analizar características en relación con la adaptabilidad al trabajo, se buscó la empatía entre los estudiantes, el liderazgo y el trabajo en colectivo.

En el segundo eje temático se estudió el papel de los contextos en la modelación matemática. Para propiciar dicha discusión se retomaron las tareas de modelación que realizó cada grupo de trabajo de manera asincrónica en documentos de Google Drive. Los docentes estuvieron en "actividad matemática", es decir, tuvieron una experiencia de creación, uso o análisis de modelos de fenómenos "reales" en la que su participación se manifestó en la exploración de ciertas estructuras subyacentes al modelo estudiado.

El tercer eje temático se relacionó con la modelación matemática y su convergencia con otras líneas de trabajo. Con el propósito de propiciar la discusión, los docentes atendieron una serie de preguntas de una situación de crecimiento fetal. La actividad se consolidó en documentos de Google Drive y los equipos hicieron uso de herramientas como el software Geogebra, chat de Google Drive, comentarios y hojas de cálculo para plantear su solución. El uso de las diferentes herramientas propició un espacio para discutir y buscar soluciones. Una de las profesoras participantes tomó el liderazgo de la discusión y para ello presentó sus aportes en tablas de Excel, que ilustraron sus ideas de solución a la situación. Su intervención generó controversia dentro del grupo: algunos apoyaron sus apreciaciones, mientras que otros presentaron argumentos para enriquecer las ideas mediante la herramienta del chat de Google Drive.

\subsection{LOS DATOS Y SU ANÁLISIS}

Para analizar la participación de los profesores se obtuvieron los datos a partir de la videograbación de la sesión sincrónica. Esta grabación fue proporcionada por la plataforma WizIQ; la grabación también aportó información relacionada con la 
participación de los estudiantes en el chat de la sesión. Otras fuentes de información fueron las anotaciones elaboradas por los estudiantes en el pizarrón, los chats de la plataforma Moodle, documentos de Google Drive y notas de campo de los investigadores. En la comunicación que sostuvieron los estudiantes a lo largo del semestre usaron también otros medios como el WhatsApp o Skype, sin embargo la información en esos medios no fue usada para este análisis.

El análisis de los datos se configuró a partir de un proceso de reducción, focalización, organización e interpretación de los mismos. La ruta de análisis comenzó con la recolección y organización de los datos en matrices. Continuó con la codificación de los diálogos y las conversaciones, de acuerdo con las manifestaciones de la participación. Enseguida, fueron contrastados los referentes conceptuales con las interpretaciones de los autores. Finalmente, se caracterizaron las maneras de participación de un grupo de docentes en ambientes online, las cuales permitieron reconocer como componentes de la misma: la visibilidad, la interactividad, las interacciones y las contribuciones. Para identificar tales componentes se efectuó un análisis de contenido, el cual se enfocó en datos que mostraran cómo y de qué maneras participaron los profesores. Para aumentar la confiabilidad de los resultados se realizó una triangulación de datos, de literatura de investigadores.

\section{CARACTERÍSTICAS DE LAS MANERAS DE PARTICIPACIÓN EN UN AMBIENTE ONLINE}

A continuación se presentan los resultados de acuerdo a la diversidad de características para la participación. La visibilidad se determinó a partir de la intensidad y el compromiso para realizar intervenciones. La interactividad, en términos de la relación con los medios tecnológicos y las posibilidades de acción y diálogo con los contenidos y los recursos. Las interacciones en correspondencia con las relaciones sociales y la acción comunicativa que medió el proceso formativo, y las contribuciones de acuerdo con los aportes, los argumentos y las apropiaciones acerca de la modelación matemática y su formación como profesor.

\subsection{LA VISIBILIDAD}

La visibilidad fue necesaria para caracterizar la participación. Por su naturaleza no pudo desligarse de las demás componentes, es decir, en las interacciones, la 
interactividad y las contribuciones los participantes también se hicieron visibles. Para dar cuenta de las características de la visibilidad, la atención se centró en su contenido (Malinen, 2015). La visibilidad se reconoció cuando los estudiantes reaccionaron a los comentarios o preguntas que formularon los orientadores o participantes de la sesión. Asimismo, cuando solicitaron ampliación o aclaración de alguna temática, o formularon cuestionamientos y comentarios articulados a la temática.

La visibilidad se observó también cuando los participantes solo se hacían notar en el chat a través de palabras como "hola", "sí", "no", "de acuerdo", pero no hubo aportes de la temática desarrollada; a esta manera de hacerse visible se le denominó simple. Otras dos maneras de visibilidad fueron identificadas: visibilidad como ampliación y visibilidad como aporte de contenido. En la Tabla $1^{2}$ para cada una de las características de la visibilidad se presenta una descripción, un ejemplo y una valoración de las veces (presencia-Pres) que ocurrió en la sesión.

La visibilidad simple fue una característica recurrente en varios de los participantes (Tatiana, Dilan, Paty, Lilo y Orlando). Al reconocerla, se implementaron tres estrategias para promover el paso de una visibilidad simple a una con aporte de contenido.

La primera de las estrategias estuvo enfocada en asignar un rol dinamizador a una de las orientadoras de la sesión (Andrew). La dinamizadora preguntaba a los participantes con visibilidad simple acerca de su presencia en la sesión, sus opiniones o conocimientos del contenido que se trabajaba. Por ejemplo, Andrew llama la atención de Paty, quien en la primera parte de la sesión tuvo poca visibilidad, y le pregunta: "Paty está em aula? [¿Paty está en clase?]", ello genera una reacción de alerta en algunos participantes, como Dilan, que pregunta "Hola Andrew, estás Ilamando a lista?", o Andi, que comenta: "si, eso estaba pensando, Andrew toma asistencia", frente a lo que la dinamizadora contesta: "Sí Dilan", "Exacto Andi", "miramos quiénes están activos en la sesión". A partir de ese momento Paty se hace más activa y visible con el contenido desarrollado en la sesión. Lo anterior evidencia cómo a partir de la estrategia se facilita pasar de la visibilidad simple al aporte de contenido.

2 Los datos son presentados tal y como los estudiantes los manifestaron en las diferentes herramientas (chat, Drive). En este sentido, pueden aparecer errores ortográficos, de digitación entre otros. 


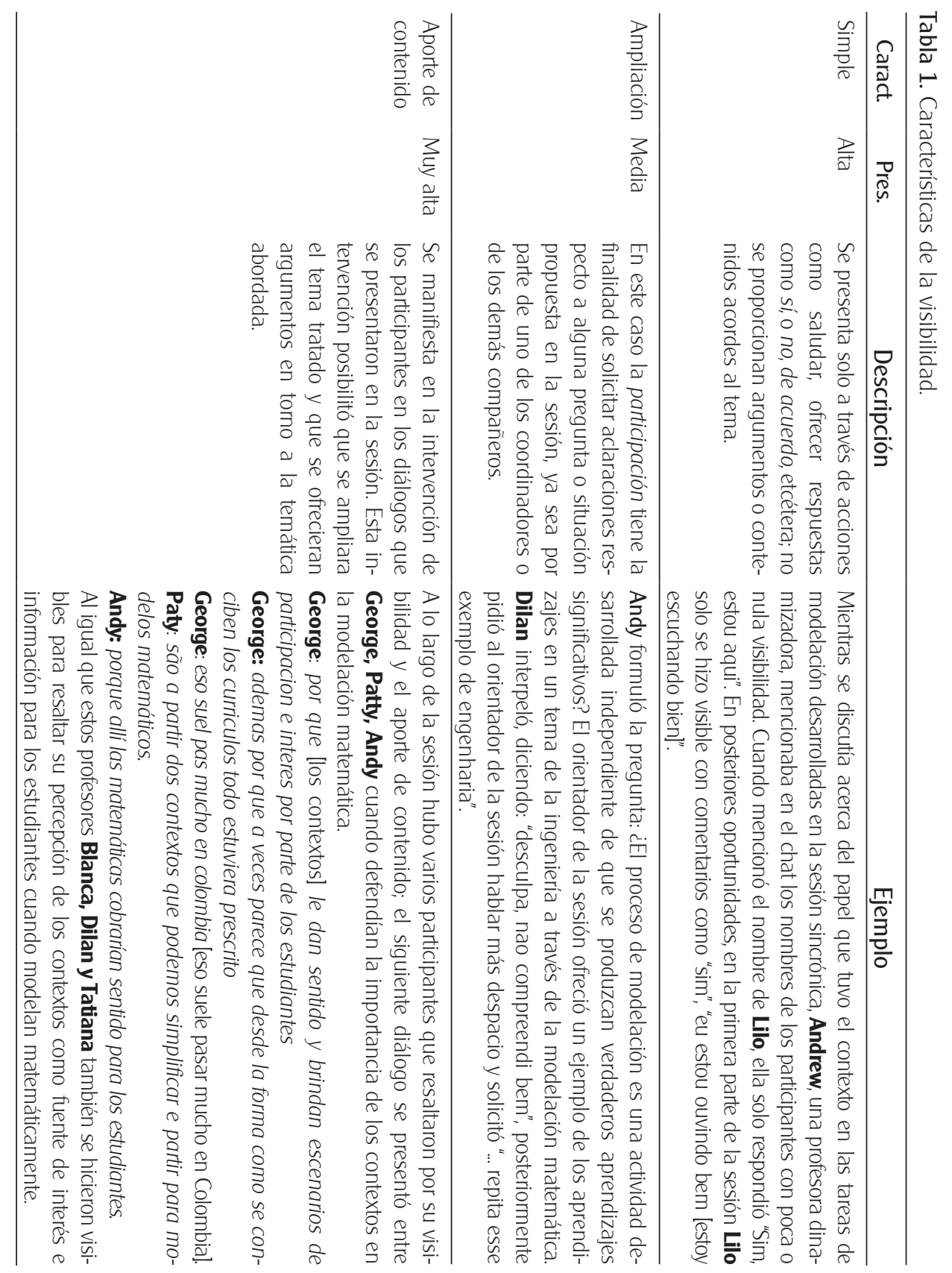


La segunda estrategia fue convocar a los estudiantes con menos visibilidad o visibilidad simple a realizar aportes en relación con la discusión, o para problematizar algo que se había dicho o hecho. La tercera estrategia consistió en promover la visibilidad a partir de las interacciones y la interactividad. El profesor orientador usó las herramientas interactivas de la plataforma para comprometer a los estudiantes con el contenido de la sesión. En diferentes momentos los orientadores trascendieron el chat y promovieron el uso de herramientas como "compartir voz", "transferir imágenes o archivos" o "escritura individual o colectiva en la pizarra", las cuales -de acuerdo con Borba et al. (2014)- promovieron la comunicación y posibilitaron que los participantes se tornaran más activos en la sesión y que, además, los compañeros pudieran visualizar, confrontar, complementar y discutir los aportes que hizo cada uno.

La visibilidad como ampliación se detectó cuando los participantes solicitaron ampliaciones o aclaraciones asociadas con los comentarios activos de los estudiantes y las explicaciones del profesor orientador; con esta visibilidad buscaron ilustrar ideas y conceptos que se proponían. A lo largo de la sesión esta visibilidad se dio como reacción a la situación que se presentaba, en la cual el estudiante actúa solamente como un receptor de la información, pero no aborda los comentarios para generar discusiones o reflexiones. Sin embargo, los profesores orientadores de la sesión, además de ofrecer las aclaraciones, cuestionaban y problematizaban sus opiniones continuamente, con el fin de promover en estos participantes una visibilidad con mayor contenido.

La visibilidad como aportes de contenido es una de las características en la que se generan intersecciones entre las componentes: visibilidad y contribuciones. Esta visibilidad ocurrió durante la sesión en momentos en los cuales los orientadores pidieron a los estudiantes que dieran respuesta a algunas preguntas planteadas para el desarrollo de las temáticas propuestas. En repetidas oportunidades los participantes respondieron a los cuestionamientos sin apoyarse o considerar las apreciaciones de los demás. Por ejemplo, al confrontar la lectura de los textos de apoyo teórico para la sesión, con las experiencias desarrolladas en la sesión previa Leidy indicó: "yo pienso que las actividades propuestas, permiten los propósitos de la modelación, ya que se dio la participación, el análisis de diversas situaciones" (imprecisiones ortográficas en el original), y Diego añadió: "Según lo que proponen los autores, la modelación permite involucrar de forma directa al estudiante". Los profesores orientadores lograron reconocer este hecho como una posibilidad de transformación del ambiente (Gerber et al., 2016) y propusieron a los participantes escribir en simultáneo en la pizarra o trabajar en Google Drive como una manera de generar interacciones entre los integrantes del colectivo. 


\subsection{LA INTERACTIVIDAD}

La interactividad es una componente de la participación en la que la relación usuario-sistema cobra especial sentido (Schultz, 2000). En este estudio se reconoció esta relación como punto y clave para promover maneras distintas de comprometer a los estudiantes en las actividades propuestas, en otras palabras, promover la participación acorde a la naturaleza online del ambiente en el que se desarrolló el curso.

En la relación usuario-sistema, más que una comprensión concatenada de la información, se tuvo en cuenta la capacidad de los participantes para intervenir en el desarrollo de las actividades. Los participantes mejoraron sus posibilidades de trabajo y de aprendizaje al proponer acciones comunicativas y actuar con diferentes recursos del entorno digital. En este estudio se lograron reconocer dos características complementarias de la interactividad: i) Actuaciones con los recursos del entorno digital, ii). Las posibilidades de comunicación. La Tabla 2 presenta una descripción, valoración de la presencia y un ejemplo para cada una de estas características.

La interactividad se reconoció en las posibilidades que ofrecen las herramientas disponibles en la plataforma y los usos educativos que se les dio; por tanto, se buscó trascender usos tecnicistas o domesticados de los medios (Borba y Villarreal, 2005). En ese sentido, las actuaciones con los recursos del entorno digital cobran un especial sentido, no solo en relación con los procesos comunicativos que se establecen, sino también con la posibilidad de aprovechar la naturaleza del ambiente online para promover aprendizajes acerca de la modelación. Un aspecto relevante es que los recursos que se habían privilegiado en las sesiones previas del curso eran: la transferencia de voz del profesor, la proyección de diapositivas en la pizarra y el chat. En el análisis de los videos de algunas sesiones previas del curso, el diseño parecía obedecer a un modelo expositivo, transmisionista y con participación poco activa de los estudiantes.

En la sesión de modelación matemática, el uso de herramientas como escribir en la pizarra y compartir voz tuvo un rol especial. Así, por ejemplo, la posibilidad de escribir directamente en la pantalla, permitió superar las limitaciones del chat en cuanto a la disponibilidad simultánea de los aportes de los participantes, la realización de análisis, confrontaciones y discusiones a la luz del contenido de cada contribución (Chang y Fisher, 2003). En la Figura 1 se observa que los participantes manipularon en la pizarra las contribuciones de sus compañeros, con el fin de relacionar las tareas de modelación estudiadas con algunas palabras clave que dieran cuenta de la interpretación de la tarea y la apropiación de los conceptos discutidos en la sesión de trabajo. 


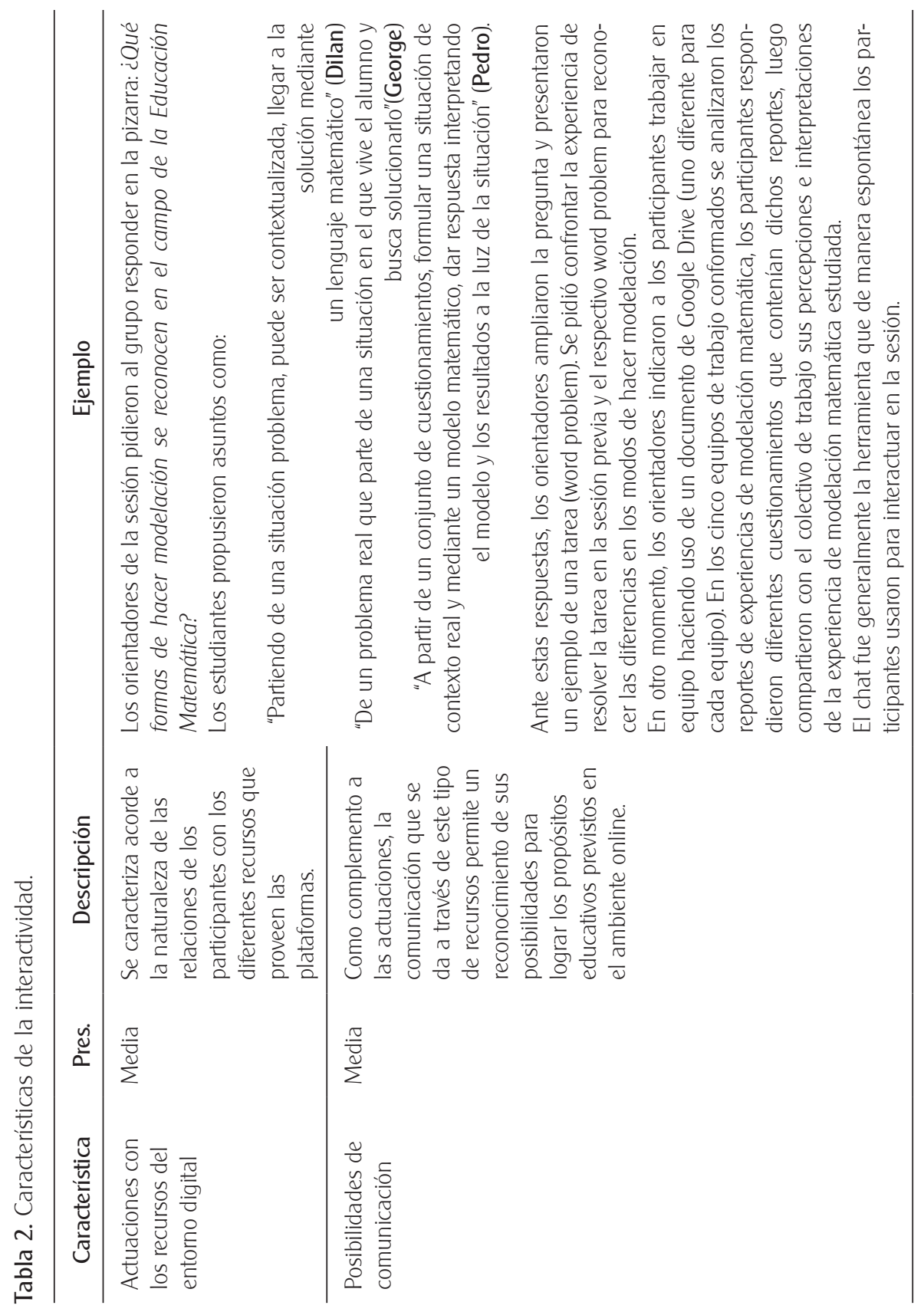




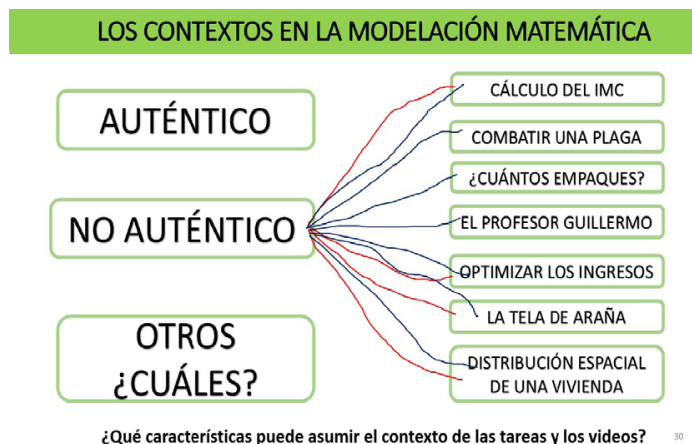

Figura 1. Relación tareas de modelación.

De acuerdo con De Wever et al. (2006) el uso y el aprovechamiento de este tipo de recursos para promover diálogo y otras interacciones es una característica de la interactividad. Dicho aprovechamiento está en coherencia con los planteamientos de Chang y Fisher (2003), quienes sugieren que en ambientes online, los estudiantes deben involucrarse en las discusiones, estar preparados para hablar, desarrollar soluciones y trabajar en colaboración, para una mayor comprensión de los conocimientos que se involucran en el ambiente.

La interactividad también se caracterizó por las posibilidades de comunicación que tuvieron lugar en el ambiente online. La comunicación dentro de la interactividad reveló un flujo bidireccional y multidireccional en los mensajes, condición que posibilitó la expresión de ideas y de conceptos involucrados en la sesión (Rost, 2004). En la sesión se pudieron reconocer las siguientes intenciones de la comunicación:

- Transmitir una información. Los participantes usaron de manera espontánea el chat para responder a las preguntas formuladas.

- Proponer debate. Se sugiere a los participantes poner en la pizarra sus opiniones y argumentos frente a cuestionamientos como ¿Qué posibilidades y limitaciones ofrece el vínculo de las matemáticas con los contextos?

- Comunicar los desarrollos. Por indicación de los orientadores, los participantes usaron las herramientas compartir pantalla, archivos y voz para dar a conocer sus respuestas y logros alcanzados en relación con las tareas propuestas. Por ejemplo, frente a la tarea de determinar si existe o no una representación algebraica para el modelo de crecimiento fetal, Sandy compartió su pantalla y acompañó de una explicación verbal la comprensión 
que realizó del modelo de crecimiento fetal con la ayuda de Excel. Además, utilizó regresiones lineales, cuadráticas y cúbicas, con las que argumentó la representación algebraica de la tarea.

La comunicación cobró sentido en el momento en que el receptor y el emisor compartieron un código para la comprensión de referentes y reformularon sus palabras habladas y escritas para poner en diálogo las percepciones de todos los participantes y consolidar significados. Otro ejemplo de esta característica de la interactividad fue percibido en el chat de la sesión sincrónica cuando los participantes ofrecieron sus reflexiones las actividades de modelación que elaboraron en colectivos de trabajo, y de la confrontación con sus posibilidades de acción en el aula.

Lidy: yo pienso que las actividades propuestas [en la sesión asincrónica], permiten los propósitos de la modelación, ya que se dio la participación, el análisis de diversas situaciones.

Dilan: Según lo que proponen los autores [que hemos leído], la modelación permite involucrar de forma directa al estudiante en su aprendizaje.

George: A mí las situaciones me parecen interesantes, sin embargo con la que nos correspondió al equipo número 4, yo no estoy muy de acuerdo con que el profesor le muestre dónde está el error usando el mismo instrumento... considero que también es importante que los profesores nos enseñen a preguntar.

Andy: precisamente este es el reto. Pienso que involucrar a los estudiantes plantea un gran reto para los profesores.

George: También quiero decir que para lograr los propósitos, dichas situaciones pueden estar aún más ajustadas a las realidades que viven los estudiantes propuestos en el proceso de modelación (Sesión 5, Chat en la plataforma WizlQ).

Como se señala en el chat, los participantes realizaron aportes y algunos se fundamentaron en las respuestas de los compañeros para dar cuenta de reflexiones, consideraciones y puntos de vista; por tanto, superaron la interactividad como una acción entre emisor-receptor, para lograr reformulaciones y realimentaciones de las ideas que posibilitan la producción y comprensión en el acto comunicativo, lo que modifica las concepciones y dinámicas de los ambientes online (Amichai-Hamburger et al., 2016). Dichas intervenciones fomentan en los participantes acuerdos y desacuerdos acerca de los temas tratados, así como la clarificación de las ideas concebidas (Gros y Silva, 2006), asunto que permite reconocer la comunicación por medio de la construcción de significados producidos conjuntamente (Schultz, 2000). 


\subsection{LAS INTERACCIONES}

Como se indicó en los referentes teóricos, las interacciones se relacionan con las discusiones, reflexiones, posturas y acciones efectuadas entre los participantes de la sesión, es decir, docentes (estudiantes de maestría) y profesores orientadores de la sesión. Las interacciones se reconocieron en aquellos episodios que generaron discusiones, reflexiones individuales y colectivas, la descripción e interpretación de ideas y el hecho de compartir puntos de vista respecto a conocimientos relacionados con la modelación matemática. A lo largo de la sesión, las interacciones se caracterizaron en las relaciones sujeto-sujeto, que se articularon con las relaciones sujeto-contenido. La Tabla 3 ofrece una descripción de cada una de ellas y proporciona ejemplos.

Al igual que en Parra-Zapata y Villa-Ochoa (2016), los participantes de este estudio manifestaron diferentes maneras de interacción, entre ellas la que se origina como una reacción natural ante las preguntas presentadas a lo largo de la sesión (interacción inducida). Enfrentarse a una tarea de modelación generó un compromiso entre diferentes grupos de trabajo que interactuaron entre sí y con el contenido para reconocer relaciones entre cantidades, realizar simplificaciones y diseñar estrategias con el propósito de determinar modelos acordes a la situación planteada. Conforme reportaron los autores, relaciones del tipo sujetos-contenido (modelación) también estuvieron presentes en un ambiente face-to-face. Sin embargo, la naturaleza online del curso descrito en este artículo requirió de maneras de interactividad complementarias para que se promoviera el trabajo en equipo, por ejemplo Google Drive y Skype.

Las principales interacciones ocurrieron en la relación estudiantes-orientadores, fundamentalmente inducidas. Pocas se dieron en la relación estudiante-estudiante, lo cual puede interpretarse como una manera de conservar el status quo del curso en general, pues en las sesiones previas a la de modelación, este tipo de interacciones fueron poco visibles. Las interacciones se caracterizaron en la relación sujeto-contenido; el contenido se refiere a todos aquellos saberes relacionados con la modelación matemática en el campo educativo. Esta característica es un punto de convergencia entre las interacciones y las contribuciones. Aunque la mayoría de las interacciones fueron dirigidas, se rescata el rol de los orientadores de la sesión en la capacidad para problematizar los avances de los participantes, lo cual promovió mayores reflexiones, orientaciones alternativas para analizar los modelos y reflexiones acerca del rol de las representaciones algebraicas en la descripción de algunos modelos matemáticos. 


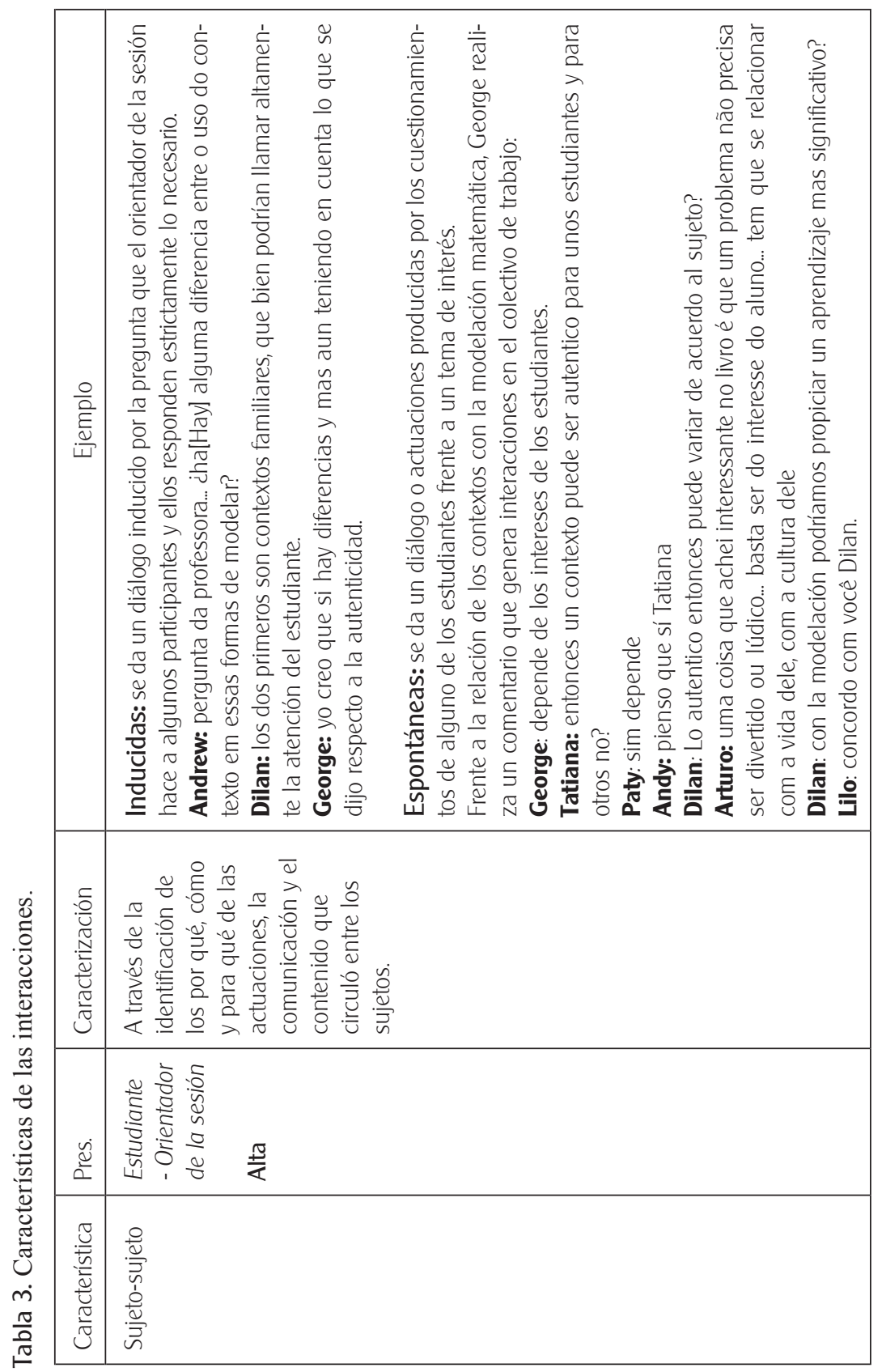




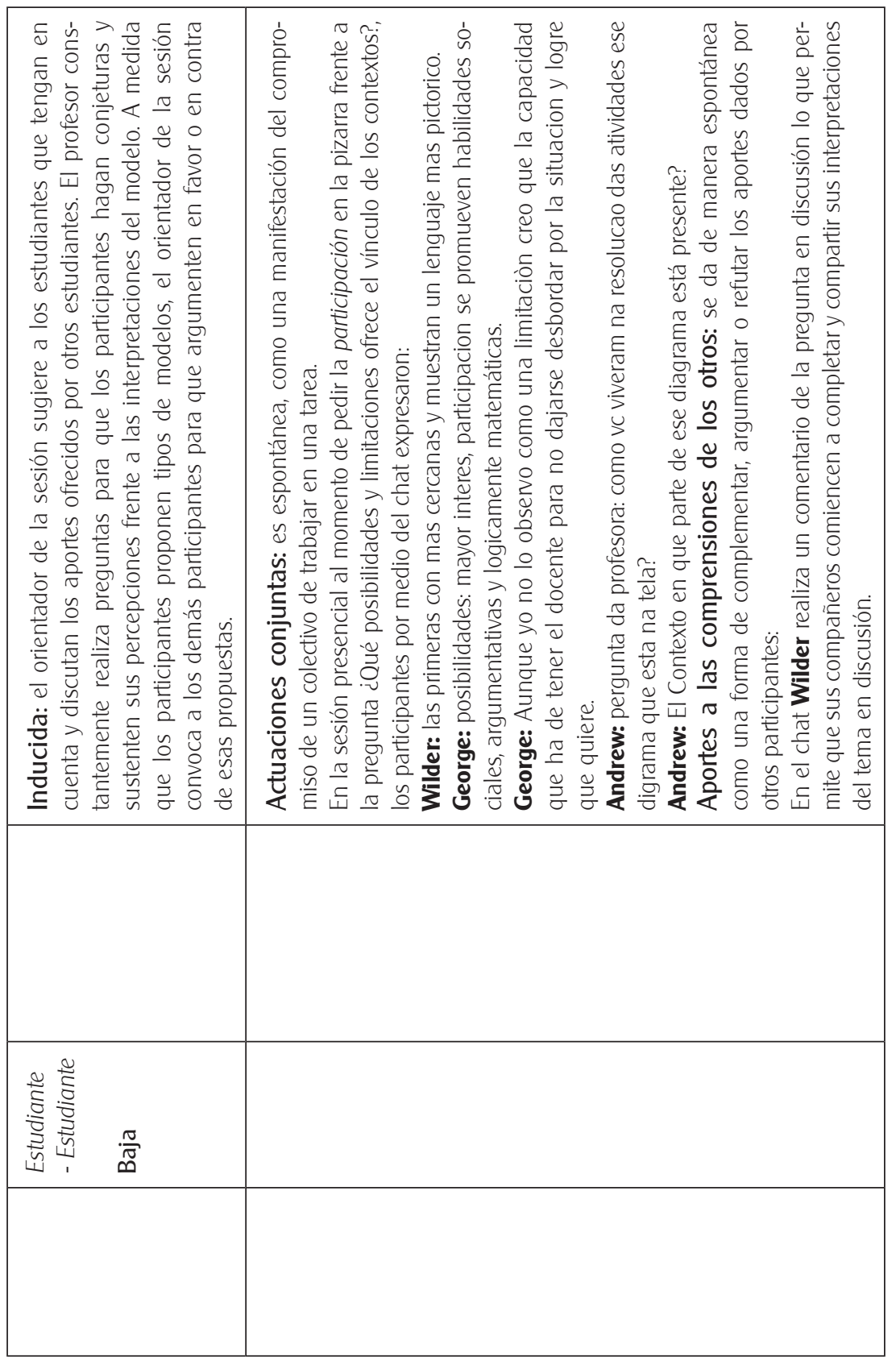




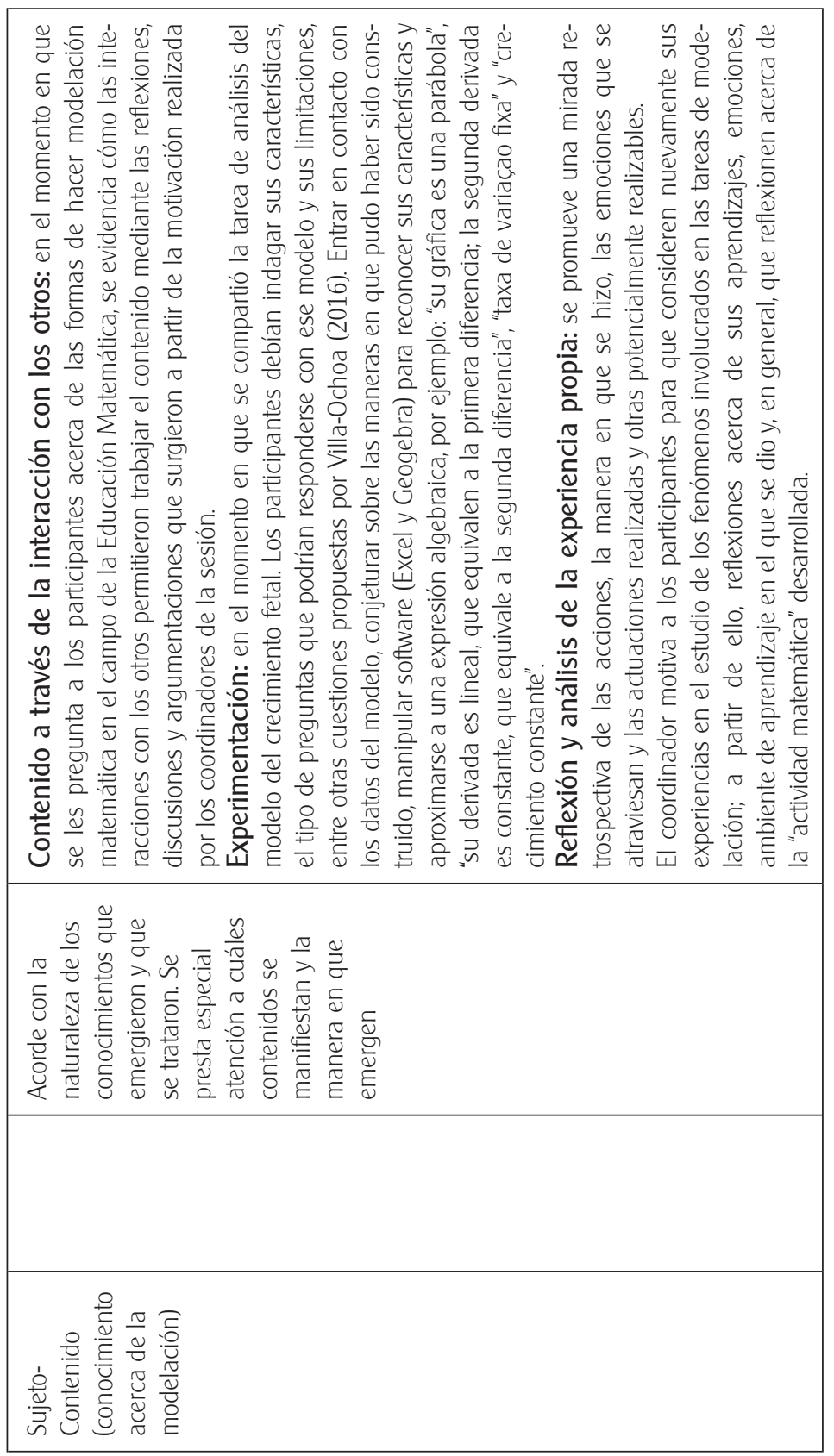




\subsection{LAS CONTRIBUCIONES}

Este apartado presenta elementos adicionales que, articulados con las demás componentes, permiten caracterizar las contribuciones para una comprensión de la participación. Las contribuciones están vinculadas con los aprendizajes y los aportes que los docentes (estudiantes de maestría) ofrecieron y reflejan sus apropiaciones del conocimiento matemático y el conocimiento en su relación con su formación como profesionales. A partir de ello, se presentan dos características adicionales de las contribuciones, a través de reflexiones frente a la importancia de la sesión para el colectivo de estudiantes, la importancia de utilizar la modelación matemática en el aula y de qué manera puede implementarse.

Las contribuciones se caracterizaron a partir de los aportes de los profesores, partiendo de su conocimiento matemático hacia alguna situación que lo requiera, es decir, en momentos puntuales en los que evidenciaron aquellas comprensiones matemáticas y habilidades para modelar los problemas que se le plantearon. En diferentes momentos de los encuentros asincrónicos y sincrónicos, los estudiantes se involucraron en el estudio de fenómenos de modelación, particularmente en la tercera parte de la sesión sincrónica se consideró una tarea que posibilitó el estudio de algunas funciones cuadráticas a partir del análisis del modelo de crecimiento fetal (Villa-Ochoa, 2016). Se pidió a los estudiantes observar los datos (Figura 2) y considerar si existía una función matemática que se ajustara al gráfico. Para responder a esta dinámica apelaron a sus conocimientos matemáticos y, en algunos casos, a software como Geogebra y Excel.

Un ejemplo de esta situación ocurrió cuando los participantes debatieron acerca de la forma de una función, de acuerdo con un modelo matemático propuesto por uno de los orientadores a partir del planteamiento de la gestación de un bebé. Sus contribuciones dieron cuenta de que su principal criterio para reconocer el tipo de modelo fue la apariencia de la gráfica. Por ejemplo, la siguiente transcripción del chat detalla algunos comentarios al respecto:

George: y no hay un decreciento del bebe.

Lilo: só considera o «dominio» positivo.

George: uno de los ejes simetricos por asi decirlo.

George: $b n$.

George: $s i$.

Andy: pode ser uma função quadratica deslocada? [¿Puede ser una función cuadrática trasladada?] 
George: no decia que a lo del dominio positivo.

George: estoy analizando la situacion.

George: los datos sobre todo.

Catherine: estou pensando.

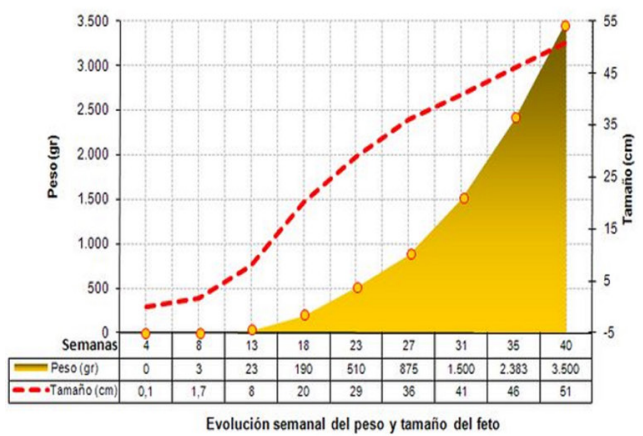

\begin{tabular}{|c|c|c|}
\hline Semanas & Peso $(\mathrm{gr})$ & Tamaño $(\mathrm{cm})$ \\
\hline 4 & 0 & 0 \\
\hline 5 & 0 & 0,1 \\
\hline 6 & 0 & 0,3 \\
\hline 7 & 0 & 1,1 \\
\hline 8 & 0 & 1,7 \\
\hline 9 & 0 & 2,4 \\
\hline 10 & 5 & 3,4 \\
\hline 11 & 10 & 4,3 \\
\hline 12 & 16 & 5,7 \\
\hline 13 & 23 & 8 \\
\hline 14 & 43 & 14 \\
\hline 15 & 70 & 15 \\
\hline 16 & 100 & 16 \\
\hline 17 & 140 & 18 \\
\hline 18 & 190 & 20 \\
\hline 19 & 240 & 22 \\
\hline 20 & 300 & 25 \\
\hline 21 & 360 & 26 \\
\hline
\end{tabular}

\begin{tabular}{|c|c|c|}
\hline Semanas & Peso $(g)$ & Tamaño \\
\hline 22 & 450 & 28 \\
\hline 23 & 510 & 29 \\
\hline 24 & 600 & 30 \\
\hline 25 & 660 & 34 \\
\hline 26 & 760 & 36 \\
\hline 27 & 875 & 37 \\
\hline 28 & 1.005 & 38 \\
\hline 29 & 1.153 & 39 \\
\hline 30 & 1.319 & 40 \\
\hline 31 & 1.500 & 41 \\
\hline 32 & 1.702 & 42 \\
\hline 33 & 1.918 & 44 \\
\hline 34 & 2.146 & 45 \\
\hline 35 & 2.383 & 46 \\
\hline 36 & 2.622 & 47 \\
\hline 37 & 2.859 & 48 \\
\hline 38 & 3.083 & 49 \\
\hline 39 & 3.288 & 50 \\
\hline 40 & 3.500 & 42 \\
\hline
\end{tabular}

Figura 2. Gráfica del crecimiento fetal. Imagen tomada de http://www.papaenapuros.com/ evolucion-del-peso-del-feto-en-el-embarazo/

En el desarrollo de la situación, la participante Sandy utilizó Excel para determinar si el modelo gráfico se ajustaba a una función cuadrática o exponencial; en ese momento, asumió un papel protagónico en la sesión. Ante sus acciones, la estudiante manifestó a través de un audio:

Inicialmente tomé una de las imágenes que estaba en la pantalla. Hice la primera gráfica y a esa gráfica se acerca a una cuadrática y todos los puntos se ajustaban a ella pero no. Eso fue lo primero que yo hice. Después volví a tomar los datos solamente hasta la semana 8. Esta fue la segunda actividad que hicimos, pero esa sí era una parábola. En tercer lugar, lo que hice fue volver a tomar los puntos pero de la semana 11 a semana 17 y con esos datos trazar la gráfica. Con herramientas de Excel reconocí la dispersión y busqué una curva que se ajustara a los puntos. La puse exponencial y se me ajustaron casi todos los puntos. No es exactamente pero se me ajusta a la mayoría de puntos. Es una gráfica muy aproximada y la expresión es $y=0,0503 e^{\wedge} 0.4749 x$

Lo anterior permitió a sus compañeros considerar otros recursos, por ejemplo Geogebra. Al respecto, Paty manifestó que: "No geogebra temos essa funcionalidade de criar cônicas por cinco pontos, mas quando seleciono o intervalo da 
9a a 13a semana, ele não identifica nenhuma quadrática possível. Encontrei essa função enquanto estávamos discutindo o primeiro exercício que vocês propuseram, foi uma forma geométrica de encontrar possíveis funçōes". Las acciones realizadas por Sandy y Paty permitieron que otros compañeros se interesaran en participar y respondieran con la utilización de otros recursos. En la manera cómo se produjo la construcción de conocimiento matemático, las contribuciones de los participantes no quedaron supeditadas a la conectividad, sino que se relacionaron con la vinculación del contenido matemático, la capacidad de discusión que posibilitaron y el papel protagónico que asumieron los estudiantes al presentar sus producciones y usar recursos de la plataforma, como la pizarra interactiva y el micrófono.

\subsection{CONCLUSIONES}

La formación en ambientes online requiere de nuevas maneras de concebir el diseño del ambiente, además de la pertinencia y la incidencia de la temática que se discuta y la producción de conocimiento matemático que suscite. A diferencia de las sesiones online convencionales, la propuesta de ambiente online en esta investigación permitió prever distintas maneras de participar, lo cual incluye el uso de diferentes recursos, herramientas y entornos, pero que posibilita una construcción y reflexión colectiva en torno al tema discutido, asunto que provoca la diferencia con otras experiencias.

Es importante mencionar que se dan nuevas connotaciones a la participación en cuanto a la visibilidad, la interactividad, las interacciones y las contribuciones. De acuerdo con estas componentes, quedó en relieve que es urgente concebir la participación en dichos escenarios y procurar que sean requisitos, además de la visibilidad y la interacción, la interactividad y las contribuciones, de tal suerte que posibiliten transformaciones en la experiencia formativa de los participantes.

Si bien la intención de la investigación era identificar y caracterizar las maneras de participación, se logró recrear cómo la participación se convirtió en el medio para desarrollar el conocimiento matemático a partir de la modelación matemática, promover discusiones que aportan al proceso formativo de los profesores (Álvarez, 2010) y cómo dichas situaciones pueden promover en los docentes nuevas visiones frente a conceptualizaciones matemáticas (Villa-Ochoa, 2016).

Los resultados sugieren que los participantes (profesores en ejercicio y estudiantes de maestría en ambientes online) deben usar su experiencia con relación 
al conocimiento para dinamizar los espacios formativos, aportar, conjeturar, contra argumentar, discutir y proponer, entre otras acciones. En este sentido, el comportamiento adaptable a las situaciones que lograron los profesores es muestra de la versatilidad a la que están dispuestos los participantes cuando se asumen comprometidos en las dinámicas formativas.

Este tipo de ambientes exige de los participantes creatividad, planificación, diseño y producción de conocimiento, entre otros elementos. En este contexto, los estudiantes pueden dejar de ser vistos como receptores de la información y, por otro lado, el papel de los participantes-coordinadores adquiere otros sentidos. El coordinador es un orientador que aporta su experiencia y conocimientos con el fin de motivar espacios de interacción y discusión entre los participantes de la sesión por medio de preguntas y le da coherencia a las discusiones, además que sintetiza los puntos de vista de todos con respecto a los temas que emergen en las discusiones de clase (Salvat y Quiroz, 2005).

Estos resultados indican una explicación de cómo las maneras en que se manifestó la participación se relacionan con las motivaciones e intereses de los estudiantes. Las acciones que efectuaron en las plataformas sugeridas y con recursos externos (Geogebra, Excel) indican que participaron en la construcción matemática asociada con la situación del control del crecimiento fetal; asimismo, relacionaron los procedimientos con otras dinámicas propuestas por el ambiente online.

Este estudio revela que la participación se manifestó a partir de la visibilidad, la interactividad, las interacciones y las contribuciones, pero no como elementos aislados, por el contrario, como componentes articulados que estructuran y dan sentido a la participación. En consonancia con Graham y Misanchuk (2004), se concluye que las maneras de participar no quedan reducidas a la cantidad o duración de la sesión ni a una participación simple de hacerse notar, sino que existen evidencias en términos de que las búsquedas propias conllevan a unas actuaciones vinculadas que posibilitan a todos los participantes (profesor y estudiantes) formar parte activa del ambiente de aprendizaje.

De acuerdo con lo anterior, la participación en ambientes online no ocurre de manera espontánea, se requiere de múltiples estrategias y de la atención de los profesores para promover maneras de participación más profundas. En particular, en este estudio se utilizaron tres estrategias: el profesor asume el rol de dinamizador durante toda la sesión, las contribuciones de los estudiantes fueron presentadas en simultáneo para discutirlas, y lo que hace el estudiante es problematizado continuamente, para promover nuevas interacciones. 


\section{REFERENCIAS}

Agyei, D. D. \& Voogt, J. (2011). ICT Use in the Teaching of Mathematics: Implications for Professional Development of Pre-service Teachers in Ghana. Education and Information Technologies, 16(4), 423-439. doi: 10.1007/s10639-010-9141-9.

Álvarez, G. (2010). Educación online: relaciones entre estructura de los cursos e intervenciones de apertura en los foros. Actualidades Investigativas en Educación, 10 (2). 1-23. doi:10.15517/aie.v16i3.26084.

Amichai-Hamburger, Y., Gazit, T., Bar-llan, J., Perez, O., Aharony, N., Bronstein, J. \& Sarah Dyne, T. (2016). Psychological Factors behind the Lack of Participation in Online Discussions. Computers in Human Behavior, 55, Part A, 268-277. doi: 10.1016/j.chb.2015.09.009.

Andrews, R. \& Haythornthwaite, C. (eds.). (2007). The SAGE Handbook of E-Learning Research. London: SAGE Publications.

Borba, M. \& Villarreal, M. (2005). Humans-with-media and the Reorganization of Mathematical Thinking: Information and Communication Technologies, Modeling, Experimentation and Visualization. New York: Springer.

Borba, M. C, Malheiros, A. P. \& Zulatto, R. (2014). Educación a distancia online (D. Pérez \& C. Tamayo, trans.). Medellín: Sello Editorial Universidad de Medellín.

Borba, M. C., Askar, P., Engelbrecht, J., Gadanidis, G., Llinares, S. \& Aguilar, M. S. (2016). Blended Learning, E-Learning and Mobile Learning in Mathematics Education. ZDMMathematics Education, 48(5), 589-610. doi: 10.1007/s11858-016-0798-4.

Chang, V. \& Fisher, D. (2003). The Validation and Application of a New Learning Environment Instrument to Evaluate Online Learning in Higher Education. In: Proceedings of the Australian Association for Research in Education Conference 2001. Australian Association for Research in Education, 1-18.

Crompton, H. (2015). Pre-service Teachers' Developing Technological Pedagogical Content Knowledge (TPACK) and Beliefs on the Use of Technology in the K-12 Mathematics Classroom: A Review of the Literature. In: C. Angeli \& N. Valanides (eds.). Technological Pedagogical Content Knowledge: Exploring, Developing, and Assessing TPCK (pp. 239-250). Boston, MA: Springer.

Danvers, G. J. (1994). -700 mots-clefs pour l'Éducation. Revue Française de Pédagogie. 107, 144-146.

De Wever, B., Schellens, T., Valcke, M. \& Van Keer, H. (2006). Content Analysis Schemes to Analyze Transcripts of Online Asynchronous Discussion Groups: A Review. Computers \& Education, 46(1), 6-28. doi: 10.1016/j.compedu.2005.04.005.

Doerr, H. \& Lesh, R. (2011). Models and Modelling Perspectives on Teaching and Learning Mathematics in the Twenty-First Century. In: G. Kaiser, W. Blum, R. Borromeo Ferri \& 
G. Stillman S. (eds.). Trends in Teaching and Learning of Mathematical Modeling (vol. 1, pp. 247-268). New York: Springer.

Fernández, C., Llinares, S. \& Valls, J. (2012). Learning to Notice Students' Mathematical Thinking through On-line Discussions. ZDM - Mathematics Education, 44(6), 747-759. doi: 10.1007/s11858-012-0425-y.

Flores, E., Escudero, D. I. \& Aguilar, M. S. (2014). Online Mathematics Teacher Education: Main Topics, Theoretical Approaches, Techniques and Changes in Researchers' Work. En: S. Oesterle, P. Liljedahl, C. Nicol \& D. Allan (eds.). Proceedings of the Joint Meeting of PME 38 and PME-NA 36 (vol. 3, pp. 89-96). Vancouver, Canadá: PME.

Galleguillos, J. E. (2016). Proposta e resoluçāo de um problema de modelagem na educação matemática online. Paper presented at the XII Encontro Nacional de Educaçāo Matemática (ENEM), Universidade Cruzeiro do Sul.

Gerber, H. R., Abrams, S. S., Curwood, J. S. \& Magnifico, A. M. (2016). Conducting Qualitative Research of Learning in Online Spaces. Thousand Oaks: SAGE Publications.

Graham, C. \& Misanchuk M. (2004). Computer-Mediated Learning Groups: Benefits and Challenges to Using Groupwork in Online Learning Environments. In: T. Roberts (ed). Online Collaborative Learning: Theory and Practice (pp. 181-202). Hershey, PA, USA: Idea Group Publishing.

Gros, B. \& Silva, J. (2006). La formación del profesorado como docentes en los espacios virtuales de aprendizaje. Revista Iberoamericana de Educación, 36(1), 1-13.

Guacaneme-Suárez, E. A., Obando-Zapata, G., Garzón, D. \& Villa-Ochoa, J. A. (2017). Colombia: Mathematics Education and the Preparation of Teachers. Consolidating a Professional and Scientific Field. In: A. Ruiz (ed.). Mathematics Teacher Preparation in Central America and the Caribbean: The Cases of Colombia, Costa Rica, the Dominican Republic and Venezuela (pp. 19-37). Switzerland: Springer.

Javaroni, S. L. \& Zampieri, M. T. (2016). Reflexōes em um espaço virtual de formaçāo de professores de matemática. Zetetiké: Revista de Educaçāo Matemática, 24(45), 108-126.

Larkin, K., Jamieson-Proctor, R. \& Finger, G. (2012). TPACK and Pre-Service Teacher Mathematics Education: Defining a Signature Pedagogy for Mathematics Education Using ICT and Based on the Metaphor "Mathematics Is a Language". Computers in the Schools, 29(1-2), 207-226.

Malinen, S. (2015). Understanding User Participation in Online Communities: A Systematic Literature Review of Empirical Studies. Computers in Human Behavior, 46, 228-238. doi: 10.1016/j.chb.2015.01.004.

Parra-Zapata, M. M. \& Villa-Ochoa, J. A. (2016). Interacciones y contribuciones. Formas de participación de estudiantes de quinto grado en ambientes de modelación 
matemática. Actualidades Investigativas en Educación, 16(3), 1-27. doi: 10.15517/aie. v16i3.26084.

Parra-Zapata, M. M., Rendón-Mesa, P. A., Molina-Toro, J. F., Sánchez-Cardona, J., Ocampo-Arenas, M. C. \& Villa-Ochoa, J. A. (2017). Participación de profesores en un ambiente de formación online. Ejemplo de un diseño en modelación matemática. Revista Virtual Universidad Católica del Norte, 50, 3-20.

Rendón-Mesa, P. A., Molina-Toro, J. F., Mesa, Y. M. \& Villa-Ochoa, J. A. (2016). Una experiencia de formación de profesores en modelación matemática en entornos mixtos de aprendizaje. En: E. Mariscal (ed.). Acta Latinoamericana de Matemática Educativa (vol. 29, pp. 958-966). México: Comité Latinoamericano de Matemática Educativa.

Rost, A. (2004). Pero, ¿̇de qué hablamos cuando hablamos de interactividad? Documento presentado al Congreso ALAIC/IBERCOM. La Plata -Argentina. 12 al 15 de octubre de 2004.

Sacristán, A. I., Parada, S. E. \& Olvera, M. (2013). Un espacio de colaboración, intercambio y experimentación en línea: conformación de comunidades de práctica y de exploraciones matemáticas a distancia. En: T. Rojano (ed.). Las tecnologías digitales en la enseñanza de las matemáticas (pp. 149-178). México: Trillas.

Salvat, B. G. \& Quiroz, J. E. S. (2005). La formación del profesorado como docente en los espacios virtuales de aprendizaje. Revista Iberoamericana de Educación, 36(1), 1-14.

Schultz, T. (2000). Interactive Options in Online Journalism: A Content Analysis of 100 US Newspapers. Journal of Computer Mediated Communication, 5(1).

Silverman, J. (2012). Exploring the Relationship between Teachers Prominence in Online Collaboration and the Development of Mathematical Content Knowledge for Teaching. Journal of Technology and Teacher Education, 20(1), 47-69.

Souto, D. L. P. \& Borba, M. C. (2016). Seres humanos-com-Internet ou Internet-com-seres humanos: uma troca de papéis? Relime-Revista Latinoamericana de Investigación en Matemática Educativa, 19(2), 217- 242. doi: 10.12802/relime.13.1924.

Villa-Ochoa, J. A. (2016). Aspectos de la modelación matemática en el aula de clase. El análisis de modelos como un ejemplo. En: J. Arrieta \& L. Díaz (eds.). Investigaciones latinoamericanas en modelación matemática educativa (pp. 109-138). Barcelona: Gedisa.

Villa-Ochoa, J. A., Galvis, J., Sierra, R. D. \& Vélez, L. Y. (2014). Integración de tecnologías en el aula de clase. El caso de los profesores implicados en el proyecto Teso. En: A. Richit (ed.). Tecnologías digitais em educaçāo: perspectivas teóricas e metodológicas sobre formaçāo e prática docente (pp. 35-56). Curitiba: Editora CRV. 